\title{
Positive Mental Imagery and Mental Health amongst University Students in Pakistan
}

\author{
Akhtar Bibi, Simon E. Blackwell, Jürgen Margraf \\ Mental Health Research and Treatment Center, Faculty of Psychology, Ruhr-Universität \\ Bochum, Germany \\ PREPRINT: PLEASE NOTE THAT THIS IS A PREPRINT VERSION OF AN \\ UNPUBLISHED MANUSCRIPT

\section{Author note:}

Akhtar Bibi, https://orcid.org/0000-0003-1494-6262

Simon E. Blackwell, https://orcid.org/0000-0002-3313-7084

Jürgen Margraf, https://orcid.org/0000-0001-5207-7016

The datasets generated and analysed during the current study are available via the Open Science Framework, https://osf.io/9hbmk/. Materials are available from the corresponding author on request.

Akhtar Bibi was supported by HEC/DAAD. The funders had no role in the design of the study, collection, analysis, and interpretation of data, or in the writing of the manuscript. We have no known conflict of interest to declare.

Correspondence concerning this article should be sent to Akhtar Bibi, Mental Health Research and Treatment Center, Faculty of Psychology, Ruhr-Universität Bochum, Massenbergstraße 9-13, 44787 Bochum, Germany. Email: akhtar.bibi@ rub.de 
PREPRINT: POSITIVE MENTAL IMAGERY IN PAKISTAN

\begin{abstract}
Objective: Mental health problems amongst university students pose a major public health challenge, and is particularly the case in Pakistan. Alongside broader societal and cultural pressures, cognitive factors likely also play a role in the development of and resilience to mental health problems and may provide a feasible target for interventions. The current study built on previous research in primarily European samples investigating the relationship between one cognitive factor, positive future-oriented mental imagery, and mental health, and aimed to extending this to university students in Pakistan.
\end{abstract}

Method: The study used a cross-sectional design, with a sample of university students in Pakistan $(N=1838)$ completing measures of mental imagery and mental health either on paper $(N=1430)$ or online $(N=408)$.

Results: Higher vividness of positive future-oriented mental imagery was associated with lower levels of depression symptoms and higher levels of positive mental health. In the sample completing the measures on paper, these relationships remained statistically significant even when controlling for socio-demographic and mental health-related variables.

Conclusion: The results provide a foundation for further investigating positive mental imagery as a potential mechanism of mental health and intervention target amongst university students in Pakistan.

Keywords: mental imagery, depression, students, positive mental health, Pakistan 


\section{PREPRINT: POSITIVE MENTAL IMAGERY IN PAKISTAN}

\section{Positive Mental Imagery and Mental Health amongst University Students in Pakistan}

There is growing evidence for a high prevalence of mental health problems amongst Pakistani university students (Saleem, Mahmood, \& Naz, 2013). A number of different factors such as academic stress, political instability, and financial constraints make these students a particularly vulnerable group for developing mental health issues. Recent research has highlighted the scale of this problem via cross-cultural comparison studies. For example, Pakistani students have higher symptoms of depression, stress and anxiety (Bibi, Lin, Zhang \& Margraf, 2020), and lower positive mental health, life satisfaction, and social support (Bibi, Lin, \& Margraf, 2020) compared to German students. Further, in comparison to German and Chinese students Pakistani students report a higher level of symptoms of mental disorders, a higher incidence of bullying in the workplace, and higher levels of suicidality, but are less likely to have received appropriate treatment (Bibi, Blackwell, \& Margraf, 2019).

In this context it can be useful to identify cognitive factors that may be protective for mental health, as these may provide a useful target for preventive interventions; although what may ultimately be needed are societal changes, more effective mental health policies and better treatment provision, in the meantime simple interventions that can provide relief in the less distant future could be hugely valuable. One potentially relevant cognitive factor relates to the experience of mental imagery, in particular positive mental imagery of possible future events. Simulation of possible positive future events via mental imagery is thought to play an important functional role in daily life, for example in planning, decision-making, and motivating goal-directed behaviour (e.g. Schacter et al., 2008; Szpunar et al., 2014). Individual differences in the experience of futureoriented imagery have been associated both with depression and with positive aspects of mental health. For example, people experiencing symptoms of depression tend to generate less vivid 
PREPRINT: POSITIVE MENTAL IMAGERY IN PAKISTAN

positive future imagery than non-depressed individuals (Holmes et al., 2016), whereas higher levels of optimism have been associated with more vivid positive future imagery (Blackwell et al., 2013; Ji et al., 2017). Although a causal role for imagery vividness in mental health has not yet been demonstrated, this seems a plausible hypothesis. For example, if someone can only dimly imagine positive events yet very vividly imagine negative events when they think about the future, this could make them feel less optimistic, more pessimistic, more hopeless, and more anxious. Positive imagery may also provide a suitable target for low-intensity interventions amongst Pakistani students, with one pilot study suggesting promise for a computerized positive imagery intervention in increasing positive imagery vividness and reducing depression symptoms (Bibi et al., 2020).

The present research therefore aimed to investigate the relationship between positive prospective imagery and mental health within a Pakistani student sample, building on and replicating results found in previous (primarily European) samples. While there was no a priori reason not to expect similar associations within a Pakistani sample, cross-cultural transferability of such findings cannot be assumed, particularly given limited cross-cultural research on mental imagery within clinical psychology (Blackwell, 2021). We used a cross-sectional dataset from 1841 university students in Pakistan that included measures of mental imagery as well as different aspects of mental health and socio-demographic variables. We aimed to investigate the relationship between positive mental imagery and both depression and positive mental health ${ }^{1}$, hypothesizing that more vivid positive prospective imagery would be associated with lower levels of depression

\footnotetext{
${ }^{1}$ We originally planned to use the Life Orientation Test-Revised (Scheier et al., 1994), a measure of optimism, as our positive mental health-related variable, as per Blackwell et al. (2013) and Ji et al. (2017), but the internal consistency in our sample was low (Cronbach's alpha $=.43$ online, .55 offline), and hence we did not analyse it and examined a positive mental health scale instead.
} 
PREPRINT: POSITIVE MENTAL IMAGERY IN PAKISTAN

and more positive mental health, including when controlling for additional potential confounding variables.

\section{Methods}

\section{Participants}

Data for the current study were collected within the Bochum Optimism and Mental Health study program (BOOM), intended to explore protective factors for positive and negative mental health. University students in Pakistan were recruited from different universities of Pakistan through online and offline sources (see Bibi, Lin, Zhang \& Margraf, 2020; Bibi, Lin \& Magraf, 2020). Participants were required to be a student aged 18-50 and fluent in English. Ethical approval was provided by the ethics committee for the Faculty of Psychology, Ruhr University Bochum, Germany (ref: 315). All participants provided informed consent on paper or online. Two thousand university students agreed to participate in the current research, but 160 participants did not complete the questionnaires and were excluded from analyses. After excluding 3 participants who did not complete the PIT, a total sample of 1838 participants (Gender: $59.6 \%$ female, $40.4 \%$ male, $\mathrm{M}_{\mathrm{age}}=22.84, \mathrm{SD}_{\mathrm{age}}=3.01$ ) completed the entire battery of self-reported questionnaires ( $N=408$ online, $N=1430$ on paper). Participants completing measures online did so in their own time from home, whereas those completing them on paper (“offline") did so in group sessions in the university. All measures were completed in English, which is the official language used at Pakistani universities.

\section{Measures}

Prospective Imagery Test (PIT; Stöber, 2000; Holmes et al. 2008). The Prospective Imagery Test is brief self-administered inventory used to measure the vividness of prospective mental imagery vividness. It consists of 10 positive (e.g. "People you meet will like you") and 10 


\section{PREPRINT: POSITIVE MENTAL IMAGERY IN PAKISTAN}

negative ("You will be the victim of crime") description of hypothetical future scenarios.

Participants were instructed to imagine each scenario as if happening to them in near future, then rate the vividness of their image on five-point scale ranging from 1 (no image at all) to 5 (very vivid). Previous studies have found the PIT to have good internal consistency (e.g., $0.83<\alpha<0.90$; Blackwell et al., 2013). In our sample of university students, for those completing the questionnaires online, Cronbach's alpha was $\alpha=0.87$ for positive imagery vividness and $\alpha=0.77$ for negative imagery vividness. For participants completing the measures on paper, Cronbach's alpha was $\alpha=0.73$ for positive imagery vividness and $\alpha=0.69$ for negative imagery vividness.

\section{Depression Anxiety Stress Scales (short form: DASS-21; Henry \& Crawford 2005).}

The DASS-21 was used to measure participants' symptoms of depression, anxiety and stress, with each of these 3 areas represented by a 7-item subscale. Participants rated how often during the past week they had experienced the symptoms listed on a scale ranging from 0 (never) to 3 (almost always). The score for each subscale is the sum of the relevant items. Cronbach's alphas in our sample were as follows: In our online sample, depression: $\alpha=.82$, anxiety: $\alpha=.72$, stress: $\alpha=.75$. In our offline sample, depression: $\alpha=.77$, anxiety: $\alpha=.72$, stress: $\alpha=.74$.

\section{Positive Mental Health (PMH-Scale; Lukat, Margraf, Lutz, van der Veld, \& Becker,}

2016). The 9-item PMH scale was used as a measure of positive mental health. It measures positive aspects of life experiences. Participants responded on a 4-point scale ranging from 0 (do not agree) to 3 (agree). In our online sample, $\alpha=0.86$, and in our offline sample, $\alpha=0.87$.

Social support scale (F-SozU K-14; Fydrich et al., 2009). The F-SozU K-14 was used to measure participants' subjective sense of their social support. It consists of fourteen items, which participants were asked to rate on seven-point scale ranging from 1 to 7 . Higher scores indicate 
PREPRINT: POSITIVE MENTAL IMAGERY IN PAKISTAN

higher subjective social support. Cronbach's alphas in our sample were $\alpha=0.88$ (online) and $\alpha=$ 0.86 (offline).

Satisfaction with life scale (SWLS; Diener, Emmons, Larsen, Griffin, 1985). The onedimensional SWLS was used to measure participants' life satisfaction. Participants responded on 7-point scale from 1 (strongly disagree) to 7 (Strongly agree). Higher scores indicate higher life satisfaction. Cronbach's alphas in our sample were $\alpha=.73$ (online) and $\alpha=.72$ (offline).

Resilience Scale (RS-11; Schumacher, Leppert, Gunzelmann, Strauß, \& Brähler, 2005). The one-dimensional RS-11 was used to measure participants' perceived. Participants responded to items on 7-point scale ranging from 1 (Disagree) to 7 (Agree). Higher scores indicate higher resilience. Cronbach's alphas in our sample were $\alpha=.84$ (online) and $\alpha=.81$ (offline).

Health scale (EQ5D; Kind, 1996). Participants' perceived general health status of was measured using the EuroQol-5D-3L visual analogue scale. Participants responded on a Visual Analogue Scale (VAS), with scores ranging from "Best imaginable health state" (100) and "Worst imaginable health state" (0).

\section{Data Analysis}

Prior to our main data analysis, we checked whether we could combine the data collected online and offline for our analyses. To do so we tested measurement invariance using Multigroup Confirmatory Factor Analysis (Byrne, 2016) of the main measures we planned to analyses. For the PIT we could only establish configural measurement invariance (see supplementary material), which suggested that we could not combine offline and online data together. There was also a statistically significant difference in Cronbach's alpha for the two samples for both positive items $\left(\mathrm{X}^{2}(1)=78.28, \mathrm{p}<.001\right)$ and negative items $\left(\mathrm{X}^{2}(1)=12.23, \mathrm{p}<.001\right)$ of the PIT, 
PREPRINT: POSITIVE MENTAL IMAGERY IN PAKISTAN

with lower internal consistency in the offline sample. We therefore analyzed the offline and online data separately ${ }^{2}$.

To examine the relationship between positive imagery vividness and both depression and positive mental health, we conducted two separate hierarchical linear regressions, one with scores on the depression subscale of the DASS-21 as the dependent variable, and one with scores on the $\mathrm{PMH}$ as the dependent variable. Our main independent variable, vividness ratings for positive items on PIT, was entered in a first step. Other independent variables were then added in a series of further steps to see which, if any, rendered the relationship between positive PIT scores and the dependent variable no longer statistically significant (as per Ji et al., 2017). Sociodemographic variables were added in the second step, followed by negative PIT scores in a third step, measures of mental health (e.g. stress, anxiety) in a fourth step, and 'positive' measures such as resilience and life satisfaction in a fifth and final step.

Analyses were conducted in SPSS version 24, with the exception of the measurement invariance analysis, which was conducted in RStudio version (1.1.463). Data is available via https://osf.io/9hbmk/.

\section{Results}

\section{Descriptive Statistics and Zero-Order Correlations}

Table 1 and 2 shows descriptive characteristic for the offline and online sample and zero order correlation with positive items of PIT.

\section{Regressions with Depression and Positive Mental Health as Dependent Variables}

Five-step linear regressions were used to investigate the relationship between positive prospective imagery vividness and both depression and positive mental health.

\footnotetext{
${ }^{2}$ We note that if we had not checked this and simply combined the two samples the results would mirror those of the larger offline sample (see Supplementary Materials for details). 


\section{PREPRINT: POSITIVE MENTAL IMAGERY IN PAKISTAN}

Results are described in Tables 2 (offline) and 3 (online). In the offline sample, scores on positive items of the PIT remained a significant predictor of scores on the DASS-21 depression scale even when controlling for all other confounding variable. Notably, although scores on the PIT explained only a small proportion of variance in DASS-21 depression scores, in the final regression they were the strongest predictor (i.e. largest $\beta$ value) apart from scores on the other DASS-21 subscales (anxiety and stress). In the online sample, inclusion of negative imagery vividness on the PIT rendered the relationship between positive PIT scores and DASS-21 depression scores no longer statistically significant.

When scores on the PMH were the dependent variable, in the offline sample scores on positive items of the PIT were significant predictors of PMH scores even when including all other variables, and in fact were the strongest predictor (largest $\beta$ value) in the final regression. In the online sample, including the measures of social support, life satisfaction, and resilience in the final step of the regression rendered the relationship between positive items on the PIT and PMH scores no longer statistically significant.

\section{Discussion}

This study investigated the relationship between positive prospective imagery vividness and both depression and positive mental health in a sample of university students in Pakistan. Results from a sample completing the measures pen-and-paper ("offline", $N=1430$ ) and another completing the measures online $(N=408)$ showed a statistically significant negative association between positive imagery vividness and depression, and a positive association between imagery vividness and positive mental health. In the pen-and-paper sample these relationships remained statistically significant when controlling for a wide range of other variables including demographics, measures of mental health and life satisfaction and perceived social support. These 
PREPRINT: POSITIVE MENTAL IMAGERY IN PAKISTAN

results extend those found in previous populations to a Pakistani sample, and highlight the potential utility of investigating positive mental imagery as a mechanism in both negative and positive aspects of mental health.

The results in the larger pen-and-paper sample echo previous studies that have suggested very specific relationships between positive prospective imagery vividness and measures of mental health or resilience (e.g. Blackwell et al., 2013; Ji et al., 2017). That is, these relationships do not appear to simply be due to shared variance with other mental health or wellbeing-related measures, and this strengthens the suggestion that there is something specific about the imagery aspect of what is measured by the Prospective Imagery Test. It is however not possible to draw definitive conclusions as to why we did not find such strong unique associations within the online sample, for example whether this was due to differences in the samples themselves or related to the method of completing the questionnaires. Given the lack of measurement invariance on the PIT between the two samples there does appear to be differences in how the participants responded on this measure. It is possible that within the online version participants were more likely to click similar responses for similarly-valenced items (particularly as the questionnaire came at the end of a long online survey), which would be consistent with the higher internal consistency in the online sample and the negative correlation between the positive and negative subscales. This in turn would lead to the PIT being a less 'true' measure of imagery vividness. However, this is purely speculative.

The results from this study cannot in themselves provide evidence for prospective imagery playing a causal role in depression or positive mental health amongst Pakistani university students, but provide a firmer foundation for investigating the possibility of such causal relationships via experimental or interventional studies. In fact, one study has investigated a computerized positive imagery intervention amongst university students in Pakistan experiencing elevated symptoms of 


\section{PREPRINT: POSITIVE MENTAL IMAGERY IN PAKISTAN}

depression, with promising results (Bibi, Margraf, \& Blackwell, 2020). However, this was only a small pilot study and therefore in need of replication. Further, although this study and several previous ones have investigated the relationship between vividness of deliberately generated positive imagery and mental health measures, it is not clear that increasing this vividness is necessarily the best target for imagery interventions. For example, it could be that the relevant aspect of scores on the PIT reflect accessibility of positive episodic memory content and the ease with which this can be projected into the future, and it is this accessibility rather than vividness per se that should be the target of interventions. From this perspective a wider range of autobiographical memory-focussed interventions (e.g. Hitchcock et al., 2017) may be helpful. The prevalence of mental health difficulties amongst these students indicates a need for broader structural changes, but if simple and easily accessible interventions were available this would be helpful for those students currently experiencing symptoms of depression.

While the large sample size in the current study and the possibility to collect data via two different methods are strengths, the study has a number of limitations. First, although university students in themselves are an important sample to study the findings cannot necessarily be generalized to clinical or older populations in Pakistan. Second, which measures we were able to include in our regressions was determined by those included in the broader BOOM study from which this data came, and therefore not selected based on theoretical considerations of which measures would be most useful to investigate imagery specifically. Finally, these analyses were not pre-registered and as such are best considered exploratory rather than confirmatory.

In summary, this research highlights positive future-oriented imagery as an important mechanism to investigate in relation to both positive and negative aspects of mental health and thus a useful target for future research. Further, it extends previous research to a new sample and 
PREPRINT: POSITIVE MENTAL IMAGERY IN PAKISTAN

culture, university students in Pakistan, providing a basis for future research investigating mental imagery as a mechanism in mental health and interventions in a population in great need of interventions.

\section{References}

Bibi, A., Blackwell, S. E., \& Margraf, J. (2019). Mental health, suicidal ideation, and experience of bullying among university students in Pakistan. Journal of health psychology, 1359105319869819.

Bibi, A., Lin, M., \& Margraf, J. (2020). Salutogenic constructs across Pakistan and Germany: A cross sectional study. International Journal of Clinical and Health Psychology, 20(1), 1-9.

Bibi, A., Lin, M., Zhang, X. C., \& Margraf, J. (2020). Psychometric properties and measurement invariance of Depression, Anxiety and Stress Scales (DASS-21) across cultures. International Journal of Psychology.

Blackwell, S. E. (2021). Mental Imagery in the Science and Practice of Cognitive Behaviour Therapy: Past, Present, and Future Perspectives. International Journal of Cognitive Therapy, 14(1), 160-181.

Blackwell, S. E., Rius-Ottenheim, N., Schulte-van Maaren, Y. W., Carlier, I. V., Middelkoop, V. D., Zitman, F. G., ... \& Giltay, E. J. (2013). Optimism and mental imagery: A possible cognitive marker to promote well-being?. Psychiatry Research, 206(1), 56-61.

Byrne, B. M. (2016). Adaptation of assessment scales in cross-national research: Issues, guidelines, and caveats. International Perspectives in Psychology: Research, Practice, Consultation, 5, 51-65. https://doi.org/10.1037/ipp0000042

Diener, E., Emmons, R. A., Larsen, R. J., \& Griffin, S. (1985). The Satisfaction With Life Scale. Journal of Personality Assessment, 49, 71-75 


\section{PREPRINT: POSITIVE MENTAL IMAGERY IN PAKISTAN}

Fydrich, T., Sommer, G., Tydecks, S., \& Brähler, E. (2009). Fragebogen zur sozialen unterstützung (F-SozU): Ntraormierung der Kurzform (K-14). Zeitschrift für Medizinische Psychologie, 18(1), 43-48.

Gusi, N., Olivares, P. R., \& Rajendram, R. (2010). The EQ-5D health-related quality of life questionnaire. Handbook of disease burdens and quality of life measures. New York: Springer, 87-99.

Henry, J. D., \& Crawford, J. R. (2005). The short-form version of the Depression Anxiety Stress Scales (DASS-21): Construct validity and normative data in a large non-clinical sample. British Journal of Clinical Psychology, 44, 227-239. https://doi.org/10.1348/014466505X29657

Hitchcock, C., Werner-Seidler, A., Blackwell, S. E., Dalgleish, T. (2017). Autobiographical episodic memory-based training for the treatment of mood, anxiety and stress-related disorders: A systematic review and meta-analysis. Clinical Psychology Review, 52, 92107. https://doi.org/10.1016/j.cpr.2016.12.003

Holmes, E. A., Blackwell, S. E., Burnett Heyes, S., Renner, F., \& Raes, F. (2016). Mental imagery in depression: Phenomenology, potential mechanisms, and treatment implications. Annual review of clinical psychology, 12, 249-280.

Holmes, E. A., Lang, T. J., Moulds, M. L., \& Steele, A. M. (2008). Prospective and positive mental imagery deficits in dysphoria. Behaviour research and therapy, 46(8), 976-981. https://doi.org/10.1207/s15327752jpa4901_13

Ji, J. L., Holmes, E. A., \& Blackwell, S. E. (2017). Seeing light at the end of the tunnel: Positive prospective mental imagery and optimism in depression. Psychiatry research, 247, 155162. 


\section{PREPRINT: POSITIVE MENTAL IMAGERY IN PAKISTAN}

Lukat, J., Margraf, J., Lutz, R., van der Veld, W. M., \& Becker, E. S. (2016). Psychometric properties of the Positive Mental Health Scale (PMH-scale). BMC Psychology, 4(1), 8. https://doi.org/10.1186/s40359-016-0111-x

Saleem, S., Mahmood, Z., \& Naz, M. (2013). Mental Health Problems in University Students: A Prevalence Study. FWU Journal of Social Sciences, 7(2), 124-130.

Schacter, D. L., Addis, D. R., \& Buckner, R. L. (2008). Episodic simulation of future events concepts, data, and applications. New York Academy of Sciences, 1124, 39-60.

Schumacher, J., Leppert, K., Gunzelmann, T., Strauss, B., \& Brähler, E. (2005). Die Resilienzskala-Ein Fragebogen zur Erfassung der psychischen Widerstandsfähigkeit als Personmerkmal [The Resilience Scale-A questionnaire for the assessment of psychosocial stress-resistance as a personality factor]. Zeitschrift für Klinische Psychologie, Psychiatrie und Psychotherapie, 53, 16 -39.

Stöber, J. (2000). Prospective cognitions in anxiety and depression: Replication and methodological extension. Cognition \& Emotion, 14(5), 725-729.

Szpunar, K. K., Spreng, R. N., \& Schacter, D. L. (2014). A taxonomy of prospection:

Introducing an organizational framework for future-oriented cognition. Proceedings of the National Academy of Sciences, 111(52), 18414-18421. 
PREPRINT: POSITIVE MENTAL IMAGERY IN PAKISTAN

Table 1. Descriptive statistics, and zero-order correlations of PIT positive imagery vividness with other study variables in the offline and online samples.

\begin{tabular}{|c|c|c|c|c|}
\hline \multirow[t]{2}{*}{ Variable } & \multicolumn{2}{|c|}{$\begin{array}{l}\text { Offline data } \\
(N=1430)\end{array}$} & \multicolumn{2}{|c|}{$\begin{array}{l}\text { Online data } \\
(N=408)\end{array}$} \\
\hline & $\mathrm{M}(\mathrm{SD})$ or $\mathrm{N}(\%)$ & $\mathrm{r}_{0}$ & $\mathrm{M}(\mathrm{SD})$ or $\mathrm{N}(\%)$ & $\mathrm{r}_{0}$ \\
\hline PIT Positive Vividness & $35.19(6.39)$ & & $32.04(8.71)$ & 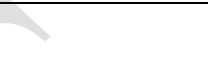 \\
\hline PMH & $17.53(6.52)$ & $.37 * * *$ & $17.04(5.59)$ & $22 * * *$ \\
\hline $\mathrm{RS}$ & $55.44(13.17)$ & $.30 * * *$ & $56.38(12.10)$ & $.19 * * *$ \\
\hline F-SozU K-14 & $50.26(11.25)$ & $.32 * * *$ & $48.94(10.23)$ & $.27 * * *$ \\
\hline SWLS & $20.34(6.50)$ & $.14 * * *$ & $20.92(5.86)$ & $.13 * *$ \\
\hline \multicolumn{5}{|c|}{ Socio-demographic variables } \\
\hline Age (years) & $22.59(2.41)$ & $.07 *$ & $23.71(4.40)$ & -.08 \\
\hline Gender, N (\%) & & 2 & & .07 \\
\hline Male & $598(41.8)$ & & $144(35.3)$ & \\
\hline Female & $832(58.2)$ & & $264(64.7)$ & \\
\hline Education, $\mathrm{N}(\%)$ & & -.01 & & .04 \\
\hline Bachelor & 94.5) & & $396(97.1)$ & \\
\hline Master or Above & $79(5.5)$ & & $12(2.9)$ & \\
\hline EQ-5D & $68.08(30.65)$ & $.18 * * *$ & $62.33(26.44)$ & $.10^{*}$ \\
\hline PIT Negative Vividness & $29.16(6.47)$ & $.10 * * *$ & $27.45(7.29)$ & $-.18 * * *$ \\
\hline \multicolumn{5}{|l|}{ DASS-21 } \\
\hline Depression & $7.79(4.35)$ & $-.15^{* * * *}$ & $7.60(4.38)$ & $-.16^{* * *}$ \\
\hline Anxiety & $8.52(4.23)$ & $-.06^{*}$ & $7.94(3.91)$ & -.07 \\
\hline Stress & $8.66(4.12)$ & -.03 & $8.19(3.70)$ & $-.13 * *$ \\
\hline
\end{tabular}

Note. $r_{0}=$ zero order correlations with PIT Positive Imagery Vividness $=$ imagery vividness ratings for positive items on the Prospective Imagery Test (PIT). PMH = Positive mental health; RS = Resilience; FSozU K-14= Social Support; SWLS = Satisfaction with life; EQ-5D = Euroqol-5D-3L (self-rated health from "Worst imaginable health state", scored as 0, to "Best imaginable health state", scored as 100); PIT Negative Imagery Vividness = imagery vividness ratings for negative items on the Prospective Imagery Test (PIT). For gender and education level, positive correlations indicate higher PIT positive scores for women and people with higher education levels, respectively. ${ }^{*} p<0.05 ;{ }^{* *} p<0.01 ;{ }^{* * *} p<0.001$. 
PREPRINT: POSITIVE MENTAL IMAGERY IN PAKISTAN

Table 2. Regression analysis of depression and positive mental health (dependent variables) and positive prospective mental imagery vividness (independent variable) in the offline sample $(n=1430)$.

\begin{tabular}{|c|c|c|c|c|c|}
\hline Independent variable & Model $1 \beta$ & Model $2 \beta$ & Model $3 \beta$ & Model $4 \beta$ & Model $5 \beta$ \\
\hline \multicolumn{6}{|c|}{ Dependent Variable: Depression } \\
\hline PIT Positive Vividness & $-.152^{* * *}$ & $-.155^{* * *}$ & $-.186^{* * *}$ & $-.123^{* * *}$ & $-.088^{* * *}$ \\
\hline Gender (female) & & $.068^{* *}$ & $.089^{* * * *}$ & .018 & .020 \\
\hline Age (years) & & $.083^{* *}$ & $.106^{* * *}$ & $.044^{* *}$ & $.044^{* *}$ \\
\hline EQ-5D & & -.045 & -.023 & $-.033 *$ & -.014 \\
\hline PIT Negative Vividness & & & $.242^{* * * *}$ & $.069^{* * * *}$ & $.058^{* * *}$ \\
\hline Anxiety & & & & $.370^{* * * *}$ & $.373^{* * *}$ \\
\hline Stress & & & & $.438^{* * *}$ & $.437^{* * * *}$ \\
\hline $\mathrm{PMH}$ & & & & & $-.044^{*}$ \\
\hline $\mathrm{RS}$ & & & & & .009 \\
\hline F-SozU K-14 & & & & & $-.058^{* *}$ \\
\hline SWLS & & & & & $-.040^{*}$ \\
\hline$R^{2}$ & .023 & .038 & .094 & .623 & .631 \\
\hline Adjusted $R^{2}$ & .022 & .035 & .091 & .621 & .629 \\
\hline$\Delta R^{2}$ change & & .015 & .057 & .529 & .008 \\
\hline$F$ for $\Delta R^{2}$ & $33.56^{* * *}$ & $7.18^{* * *}$ & $88.88^{* * *}$ & $997.14^{* * *}$ & $8.157^{* * *}$ \\
\hline$F$ for model & $33.56^{* * *}$ & $13.89^{* * *}$ & $29.57^{* * *}$ & $335.57^{* * *}$ & $220.81^{* * *}$ \\
\hline
\end{tabular}

\section{Dependent variable: Positive Mental Health}

\begin{tabular}{|c|c|c|c|c|}
\hline PIT Positive Vividness $\quad .366^{* * *}$ & $.333^{* * * *}$ & $.348^{* * * *}$ & $.330^{* * * *}$ & $.211^{* * * *}$ \\
\hline Gender (female) & -.010 & -.020 & -.020 & -.043 \\
\hline Age (years) & .042 & .031 & .036 & .037 \\
\hline EQ-5D & $.179^{* * *}$ & $.168^{* * * *}$ & $.162^{* * * *}$ & $.107^{* * *}$ \\
\hline PIT Negative Vividness & & $-.119^{* * *}$ & $-.112^{* * * *}$ & $-.081^{* * *}$ \\
\hline Depression & & & $-.151^{* * *}$ & $-.081^{*}$ \\
\hline Anxiety & & & .046 & .013 \\
\hline Stress & & & $.097^{*}$ & $.073^{*}$ \\
\hline
\end{tabular}


PREPRINT: POSITIVE MENTAL IMAGERY IN PAKISTAN

\begin{tabular}{lccccc}
\hline RS & & & & & $.182^{* * *}$ \\
F-SozU K-14 & & & & & $.180^{* * *}$ \\
SWLS & & & & & $.162^{* * *}$ \\
\hline$R^{2}$ & .134 & .167 & .181 & .190 & .314 \\
Adjusted $R^{2}$ & .134 & .165 & .178 & .186 & .309 \\
$\Delta R^{2 \text { change }}$ & .134 & .033 & .014 & .009 & .124 \\
$F$ for $\Delta R^{2}$ & $221.40^{* * *}$ & $18.96^{* * *}$ & $23.73^{* * *}$ & $5.39^{* *}$ & $85.24^{* * *}$ \\
$F$ for model & $221.40^{* * *}$ & $71.66^{* * *}$ & $62.99^{* * *}$ & $41.75^{* * *}$ & $59.02^{* * *}$ \\
\hline
\end{tabular}

Note. PIT Positive Imagery Vividness $=$ imagery vividness ratings for positive items on the Prospective Imagery Test (PIT); PMH = Positive mental health; RS = Resilience; F-SozU K-14= Social Support; SWLS = Satisfaction with life; EQ-5D = Euroqol-5D-3L (self-rated health from "Worst imaginable health state", scored as 0, to "Best imaginable health state", scored as 100); PIT Negative Imagery Vividness = imagery vividness ratings for negative items on the Prospective Imagery Test (PIT). ${ }^{*} p<0.05 ;{ }^{* *} p<0.01 ;{ }^{* * *} p<0.001$. 
PREPRINT: POSITIVE MENTAL IMAGERY IN PAKISTAN

Table 3. Regression analysis of depression and positive mental health (dependent variables) and positive prospective mental imagery vividness (independent variable) in the online sample $(N=408)$.

\begin{tabular}{|c|c|c|c|c|c|}
\hline Independent variable & Model $1 \beta$ & Model $2 \beta$ & Model $3 \beta$ & Model $4 \beta$ & Model $5 \beta$ \\
\hline \multicolumn{6}{|c|}{ Dependent variable: Depression } \\
\hline PIT Positive Vividness & $-.163^{* * * *}$ & $-.110^{*}$ & -.064 & -.047 & -.019 \\
\hline Gender (female) & & $-.098^{*}$ & -.085 & -.036 & -.032 \\
\hline Age (years) & & $.173^{* * *}$ & $.181^{* * *}$ & $.125^{* * *}$ & $.092^{* *}$ \\
\hline EQ-5D & & $-.312^{* * *}$ & $-.288^{* * *}$ & $-.116^{* * *}$ & $-.084^{* *}$ \\
\hline PIT Negative Vividness & & & $.272^{* * *}$ & $.065^{*}$ & $.065^{*}$ \\
\hline Anxiety & & & & $.385^{\text {**** }}$ & $.367^{* * *}$ \\
\hline Stress & & & & $.393^{* * *}$ & $.369^{* * *}$ \\
\hline $\mathrm{PMH}$ & & & & & -.011 \\
\hline $\mathrm{RS}$ & & & & & -.048 \\
\hline F-SozU K-14 & & & & & -.050 \\
\hline SWLS & & & & & $-.102^{* *}$ \\
\hline$R^{2}$ & .027 & .173 & .244 & .676 & .699 \\
\hline Adjusted $R^{2}$ & .024 & .165 & .235 & .670 & 690 \\
\hline$\Delta R^{2}$ change & .027 & .147 & .071 & .432 & .023 \\
\hline$F$ for $\Delta R^{2}$ & $11.06^{* * *}$ & $23.86^{* * *}$ & $37.68^{* * *}$ & $266.64^{* * *}$ & $7.43^{* * *}$ \\
\hline$F$ for model & $11.06^{* * *}$ & $21.12^{* * * *}$ & $25.97^{* * *}$ & $119.25^{* * * *}$ & $83.47^{* * * *}$ \\
\hline
\end{tabular}

\section{Dependent variable: Positive Mental Health}

\begin{tabular}{|c|c|c|c|c|}
\hline PIT Positive Vividness $.223^{* * *}$ & $.189^{* * * *}$ & $.187^{* * * *}$ & $.163^{* * *}$ & .072 \\
\hline Gender (female) & -.049 & -.050 & -.075 & $-.086^{*}$ \\
\hline Age (years) & $-.143^{* *}$ & $-.144^{* *}$ & -.089 & .008 \\
\hline$E Q-5 D$ & $.253^{* * * *}$ & $.252^{* * * *}$ & $.160^{* * *}$ & .068 \\
\hline PIT Negative Vividness & & -.009 & .076 & .059 \\
\hline Depression & & & $-.281^{* * *}$ & -.018 \\
\hline Anxiety & & & .062 & .027 \\
\hline Stress & & & -.106 & $-.123^{*}$ \\
\hline
\end{tabular}


PREPRINT: POSITIVE MENTAL IMAGERY IN PAKISTAN

RS

$197^{* * *}$

F-SozU K-14

$.206^{* * *}$

SWLS

$391^{* * *}$

$R^{2}$

Adjusted $R^{2}$

$\Delta R^{2 \text { change }}$

.047

.050

.050

.139

.139

.218

.518

$F$ for $\Delta R^{2}$

$21.27^{* * * *}$

.130

.128

.202

.504

$F$ for model

$21.27^{* * *}$

.089

.000

.079

.300

Note. PIT Positive Imagery Vividness = imagery vividness ratings for positive items on the Prospective Imagery Test (PIT). PMH = Positive mental health; RS = Resilience; F-SozU K-14= Social Support;

SWLS = Satisfaction with life; EQ-5D = Euroqol-5D-3L (self-rated health from "Worst imaginable health state", scored as 0, to "Best imaginable health state", scored as 100); PIT Negative Imagery Vividness = imagery vividness ratings for negative items on the Prospective Imagery Test (PIT). ${ }^{*} p<0.05 ;{ }^{* *} p<0.01 ;{ }^{* * *} p<0.001$. 


\section{Supplementary Material}

Supplementary Table 1. Regression analysis of depression and positive mental health (dependent variables) and positive prospective mental imagery vividness (main independent variable) in total sample $(N=1838)$.

\begin{tabular}{|c|c|c|c|c|c|}
\hline Independent variable & Model $1 \beta$ & Model $2 \beta$ & Model $3 \beta$ & Model $4 \beta$ & Model $5 \beta$ \\
\hline \multicolumn{6}{|c|}{ Dependent Variable: Depression } \\
\hline PIT Positive Vividness & $-.156^{* * *}$ & $-.143^{* * *}$ & $-.151^{* * *}$ & $-.102^{* * *}$ & $-.065^{* * *}$ \\
\hline Method of completion & $.047^{*}$ & $.074^{* *}$ & $.051^{*}$ & .003 & -.003 \\
\hline Gender (female) & & .030 & $.050^{*}$ & .001 & .006 \\
\hline Age (years) & & $.124^{* * *}$ & $.139^{* * * *}$ & $.072^{* * *}$ & $.066^{* * *}$ \\
\hline EQ-5D & & $-.101^{* * *}$ & $-.081^{*}$ & $-.055^{* * *}$ & $-.030^{*}$ \\
\hline PIT Negative Vividness & & & $.243^{* * *}$ & $.063^{* * *}$ & $.054^{* * * *}$ \\
\hline Anxiety & & & & $.377^{* * *}$ & $.377^{* * *}$ \\
\hline Stress & & & & $.430^{* * *}$ & $.425^{* * *}$ \\
\hline $\mathrm{PMH}$ & & & & & $-.045^{* *}$ \\
\hline $\mathrm{RS}$ & & & & & -.006 \\
\hline F-SozU K-14 & & & & & $-.058^{* * *}$ \\
\hline SWLS & & & & & $-.051^{* *}$ \\
\hline$R^{2}$ & .024 & .050 & .107 & .631 & .642 \\
\hline Adjusted $R^{2}$ & .023 & .047 & .104 & .629 & .640 \\
\hline$\Delta R^{2}$ change & .024 & .026 & .058 & .523 & .012 \\
\hline$F$ for $\Delta R^{2}$ & $22.38^{* * *}$ & $16.63^{* * *}$ & $117.10^{* * *}$ & $1295.71^{\text {*** }}$ & $15.16^{* * *}$ \\
\hline$F$ for model & $22.38^{* * *}$ & $19.16^{* * *}$ & $36.65^{* * *}$ & $390.29^{* * *}$ & $273.30^{* * *}$ \\
\hline \multicolumn{6}{|c|}{ Dependent variable: Positive mental health } \\
\hline PIT Positive Vividness & $.330^{* * *}$ & $.298^{* * *}$ & $.301^{* * *}$ & $.279^{* * *}$ & $.163^{* * *}$ \\
\hline Method of completion & -.029 & -.041 & -.033 & -.031 & .002 \\
\hline Gender (female) & & -.007 & -.013 & -.012 & $-.043^{*}$ \\
\hline Age (years) & & -.007 & -.012 & .005 & .034 \\
\hline $\mathrm{EQ}-5 \mathrm{D}$ & & $.202^{* * *}$ & $.196^{* * *}$ & $.184^{* * *}$ & $.110^{* * * *}$ \\
\hline
\end{tabular}




\section{SUPPLEMENTARY MATERIAL: POSITIVE MENTAL IMAGERY IN PAKISTAN}

\begin{tabular}{|c|c|c|c|c|c|}
\hline PIT Negative Vividness & & & $-.081^{* * *}$ & $-.062^{* *}$ & $-.040^{*}$ \\
\hline Depression & & & & $-.194^{* * *}$ & $-.085^{* *}$ \\
\hline Anxiety & & & & .049 & .011 \\
\hline Stress & & & & $.078^{*}$ & .050 \\
\hline $\mathrm{RS}$ & & & & & $.201^{* * * *}$ \\
\hline F-SozU K-14 & & & & & $.196^{* * *}$ \\
\hline SWLS & & & & & $.201^{* * *}$ \\
\hline$R^{2}$ & .106 & .146 & .152 & .167 & .331 \\
\hline Adjusted $R^{2}$ & .105 & .143 & .149 & .163 & .327 \\
\hline$\Delta R^{2 \text { change }}$ & .106 & .040 & .006 & .015 & .165 \\
\hline$F$ for $\Delta R^{2}$ & $108.65^{* * *}$ & $28.42^{* * *}$ & $13.87^{* * *}$ & $10.65^{* * *}$ & $149.87^{* * *}$ \\
\hline$F$ for model & $108.65^{* * *}$ & $62.46^{* * *}$ & $54.73^{* * *}$ & $40.61^{* * *}$ & $75.37^{* * *}$ \\
\hline
\end{tabular}

Note. PIT Positive Imagery Vividness = imagery vividness ratings for positive items on the Prospective Imagery Test (PIT); Method of completion = online (=1) vs. offline (=2); PMH = Positive mental health; RS = Resilience; F-SozU K-14= Social Support; SWLS = Satisfaction with life; EQ-5D = Euroqol-5D-3L (self-rated health from "Worst imaginable health state", scored as 0, to "Best imaginable health state", scored as 100); PIT Negative Imagery Vividness = imagery vividness ratings for negative items on the Prospective Imagery Test (PIT). ${ }^{*} p<0.05 ;{ }^{* *} p<0.01 ;{ }^{* * *} p<0.001$. 


\section{SUPPLEMENTARY MATERIAL: POSITIVE MENTAL IMAGERY IN PAKISTAN}

Supplementary Table 2. Measurement Invariance of Prospective Imagery Test (PIT)

\begin{tabular}{|c|c|c|c|c|c|c|c|c|}
\hline Models & $N$ & Chi2/df & RMSEA & 90\% C.I. & CFI & TLI & SRMR & $\Delta \mathrm{CFI}$ \\
\hline \multicolumn{9}{|c|}{ Single group CFA-Original one factor model of Positive PIT } \\
\hline offline & 411 & $252.828 / 35$ & .066 & $.058-.074$ & .883 & .850 & .044 & \\
\hline online & 14 & $67.202 / 35$ & .047 & $.030-.064$ & .977 & .970 & .032 & \\
\hline \multicolumn{9}{|c|}{ Multiple group CFA. } \\
\hline Configura & & $\begin{array}{l}320- \\
030 / 70\end{array}$ & .062 & $.056-.069$ & .923 & .901 & .038 & \\
\hline Weak & & $371.156 / 79$ & .063 & $.057-.070$ & .910 & .898 & .050 & .013 \\
\hline Strong & & $409.981 / 88$ & .063 & $.057-.069$ & .901 & .899 & .052 & .022 \\
\hline
\end{tabular}

\begin{tabular}{|c|c|c|c|c|c|c|c|c|}
\hline Models & $N$ & Chi2/df & RMSEA & 90\% C.I. & CFI & TLI & SRMR & $\Delta \mathrm{CFI}$ \\
\hline \multicolumn{9}{|c|}{ Single group CFA-Original one factor model of PIT negative } \\
\hline offline & 1841 & $436.909 / 35$ & .090 & $.082-.097$ & .765 & .698 & .065 & \\
\hline online & 7721 & $150.777 / 35$ & .090 & $.076-.105$ & .847 & .803 & .070 & \\
\hline \multicolumn{9}{|c|}{ Multiple group CFA. } \\
\hline Configura & & $587.686 / 70$ & .090 & $.083-.096$ & .790 & .730 & .061 & \\
\hline Weak & & $599.255 / 79$ & .085 & $.078-.091$ & .789 & .760 & .062 & \\
\hline Strong & & $409.981 / 88$ & .063 & $.057-.069$ & .901 & .899 & .052 & \\
\hline
\end{tabular}

